\title{
La Gestión Cultural en Costa Rica: definiciones y alcances
}

\author{
Cultural Management in Costa Rica: definition and scope \\ Nicolás F. Alpízar Valverde ${ }^{1}$
}

Fecha de recepción: 27-8-2019

Fecha de aprobación: 26-11-2019

\begin{abstract}
Resumen
Este texto presenta un marco descriptivo y conceptual específico para la gestión cultural contemporánea en Costa Rica. Para ello elabora una revisión crítica de conceptos, un recuento histórico y una propuesta de nomenclatura; todo acompañado por parte de una consulta empírica. Inicia con la revisión de las dos vertientes conceptuales de la Gestión Cultural. Primero se reseña la evolución desde la administración clásica hacia la gestión, de carácter más contemporáneo. A continuación, dada la extensa discusión sobre el concepto "cultura", se contraponen dos acepciones, una restringida y una amplia, las cuales coexisten tanto en las convenciones internacionales como en la praxis cotidiana. Para subsanar ambigüedades, se propone una categorización de los bienes culturales, en vez del uso tradicional de "patrimonio cultural". Posteriormente, se analiza la gestión cultural costarricense desde la teoría, acompañada por una reseña historiográfica. Gracias a la consulta directa con personas que la ejercen, se describen los objetos y las prácticas que los profesionales ejercen en suelo nacional. El autor finaliza con sus reflexiones y, lejos de emitir criterios absolutos, espera propiciar la discusión e incentivar futuras investigaciones.
\end{abstract}

Palabras clave: Gestión Cultura, Costa Rica, Definiciones y alcances, Historia, Políticas culturales.

\begin{abstract}
This text presents a specific descriptive and conceptual framework for contemporary Cultural Management in Costa Rica. To achieve that, it develops a critical review of concepts, a historical overview and a reclassification proposal; along by an empirical consultation. It begins with the review of the two conceptual aspects of Cultural Management. At the beginning, the evolution from classical administration to more contemporary management is reviewed. Then, given the extensive discussion on the concept of Culture, two meanings are contrasted, one restricted and one broad, which coexist both in international conventions and in daily practice. In order of clearing ambiguities, a categorization of cultural assets is proposed, instead of the traditional use of Cultural Heritage. Subsequently, it analyzes the Costa Rican Cultural Management from the theory and through a historiographic revision. Due to direct consultation with people who practice it, the objects and practices that professionals practice on national land are described. The author ends with his reflections and, far from issuing closed criteria, he hopes to promote discussion and encourage future research.
\end{abstract}

Keywords: Cultural Management - Costa Rica - Definition and scope - History - Cultural policies.

1 Licenciado en Artes Plásticas, Universidad de Costa Rica. Máster en Administración de Negocios, Universidad Estatal a Distancia de Costa Rica. Coordinador del Bachillerato en Gestión Cultural de la Universidad de Costa Rica, Costa Rica. Correo electrónico: nicoli.alpizarvalverde@ ucr.a.cr 


\section{Introducción}

En 2013, en la Sede del Pacífico de la Universidad de Costa Rica, surgió la inquietud de crear una carrera en Gestión Cultural. Dado que existían ofertas académicas y prácticas profesionales similares en otras latitudes, se impuso desde el inicio una interrogante: ¿cuál es la forma presente de la Gestión Cultural en Costa Rica? Esta pregunta se convirtió en un amplio proyecto de búsqueda y en el objetivo principal de este artículo. El tema cuenta con una muy limitada bibliografía; a pesar de lo extendido de la práctica en suelo costarricense. Se hizo indispensable crear un parámetro teórico y conceptual que pudiera sentar una base descriptiva a priori. Fue necesario también comenzar con definiciones internacionales que han sido tamizadas a través de algunos estudios nacionales y enriquecidas con trabajo de campo.

Este trabajo pretende describir la forma que toma la Gestión Cultural en Costa Rica desde los enfoques teórico, histórico y epistemológico, con el fin de incentivar futuras discusiones. Para ello, se inicia con una aclaratoria del concepto de "administración", el cual ha ido evolucionando hasta convertirse en el de "gestión". También, se contraponen las dos nociones más contemporáneas sobre cultura, que coexisten en suelo nacional. Con ello, se establece una idea de Gestión Cultural acorde con la realidad costarricense, la cual permite determinar los objetivos y los campos de acción.

El progreso de las políticas culturales nacionales e internacionales de los últimos cincuenta años, ha modificado el destino de los servicios culturales; así como la gestión. Esto ha decantado en un consenso sobre el nacimiento de la Gestión Cultural entre los costarricenses que se relacionan directamente con el tema; caso contrario, el de las personas ajenas, para quienes el concepto resulta aún poco claro. Con este escrito, se espera facilitar un lenguaje común para los especialistas; así como una mayor claridad en el área en todos los niveles, lo cual permite el reconocimiento de una profesión especializada, interdisciplinaria y compleja, ante las instancias oficiales y el público en general.

\section{Metodología}

Este es un estudio de enfoque cualitativo. Dada la escasez de publicaciones originales sobre el consumo y la gestión de la cultura en Costa Rica, tiene un alcance exploratorio y descriptivo. Se ejecutó una búsqueda bibliográfica de los conceptos que permiten el surgimiento de la Gestión Cultural como práctica profesional específica y diferenciada. La mayoría del material fue ubicado mediante rastreo digital, consistió en libros de texto, artículos y memorias. Entre los autores nacionales más destacados se hallan Muñoz (2009), Monge (2011), González y Hernández (2012), Rodríguez y Protti (2009); así como el propio Ministerio de Cultura y Juventud de Costa Rica (2013; 2016). Respecto de las fuentes internacionales, sobresalen Martínez (1997), Martinell (2001) y la Organización de las Naciones Unidas para la Educación, la Ciencia y la Cultura (UNESCO, 1972; 2003).

Los datos acerca de las prácticas y los objetos de la Gestión Cultural corresponden con un segmento del estudio de viabilidad para el Bachillerato en Gestión Cultural en la Sede del Pacífico de la Universidad de Costa Rica (Alpízar, 2014), en el cual se realizó una consulta con gestores culturales en ejercicio. El examen de los datos se hizo mediante inmersión profunda y análisis de contenido. Específicamente, para la manufactura de los objetos material y formal, se utilizó la metodología propuesta por el Centro de Evaluación Académica de la Universidad de Costa Rica (Bolaños, s.f.). Los resultados son presentados en prosa, con el apoyo de cuadros.

\section{Construcción conceptual de la Gestión Cultural}

Gestión Cultural es un concepto que resulta de dos filosofías de pensamiento: la "gestión" como la puesta al día de la administración y la polémica sobre el significado del vocablo "cultura". En este marco, es oportuna una revisión pueda ser enfocada hacia la realidad costarricense. 


\subsection{De la administración clásica a la gestión}

Drucker (citado por Martinell, 2001) define tres períodos del modelo capitalista: "revolución Industrial" (1750-1850), "revolución de la productividad" (1850-1950) y "revolución de la gestión” (1950-1980). La primera se caracterizó por el desarrollo de tecnologías basadas en el uso de combustibles fósiles, el hacinamiento de la gente en grandes ciudades y el poder ostentado por comerciantes e industriales. El modelo gobernante era la explotación del trabajador y el comercio internacional de mercancías, basados en la balanza comercial y especialización internacional del trabajo. A pesar de ello, la industria y la manufactura a gran escala se afianzaron, entonces surgió la brecha entre el productor y el consumidor como estructura económica básica. En la segunda, Taylor dio a conocer los preceptos científicos de la administración, los cuales se enfocaron en el estudio del trabajo. A posteriori, surgieron grupos centrados en aumentar el nivel de productividad en las organizaciones y el consumo de bienes. La empresa se concebía mecánica, rígida y lógica. Esta tónica de mando se afianzó en los años cincuenta, con el establecimiento del consumismo como fórmula económica (Chiavenato, 2006). En la tercera, se cuestionaron los valores mecanicistas, sustituyéndolos por procesos y objetivos más amplios que la producción. Los nuevos enfoques eran ahora más cercanos al bienestar y las relaciones sociales. Se incluyeron el ámbito terciario y los servicios como entes de desarrollo, junto con un renovado cuido de los capitales intangibles (Drucker, 1994).

Unido a lo anterior, la gestión es (...) un lenguaje complejo que nos aleja de la estricta casualidad de lo factual o la rutina del mantenimiento, y nos acerca mucho más al concepto de política (entendida como preferencia). Reclama una capacidad de definir objetivos y de diseñar el proyecto como eje y metodología de los actos. Exige un cierto gusto por la autonomía para decidir el curso de los hechos y la libertad para resolver los problemas que emergen a la hora de ejecutar (Martinell, 2007).

En la actualidad es posible distinguir dos tipos generales de estructuras organizacionales: mecanicistas y orgánicas (Franklin y Krieger, 2011). Las mecanicistas se hallan ancladas en el modelo de la Revolución de la Administración y se concentran en la jerarquía de autoridad, las reglas, la centralización y los procedimientos. Del otro lado, están las organizaciones orgánicas, también llamadas "de la complejidad" por Martinell; son de origen más reciente y encajan con el modelo de gestión. De esta manera, se hace cada vez más frecuente el uso de "gestor", en vez de "administrador" o "gerente".

\subsection{Distintas definiciones para "cultura"}

La palabra "cultura" ha tenido una enorme cantidad de usos, los cuales van desde lo popular a lo elitista, desde lo literario a lo científico. Por lo que, en el panorama cultural costarricense, se presenta el mismo debate. Irene Martínez (1997) elabora un valioso análisis semiótico e historial sobre el término. Según dicha autora, existen dos acepciones principales y opuestas entre sí: la primera es restringida y excluyente; la segunda es abierta e inclusiva (cuadro 1). Señala que "cultura" tiene el origen en la voz latina "cultus", cuyos significados originales son "de realizaciones intelectuales y artísticas", las cuales deben ser desarrolladas o cultivadas; así como la capacidad para comprender y disfrutar de ellas. De esta manera, se puede "ser culto" y "tener cultura". Este sentido se relaciona con la idea de "civilizar", y dicho sentido fue impulsado desde la Ilustración por franceses e ingleses. Como reacción contraria, Martínez apunta que en la Alemania romancista surgió una corriente (iniciada por Rousseau) que criticaba esta visión: "El progreso de las artes y las ciencias, lejos de perfeccionar al hombre y a la sociedad, multiplica sus vicios y lacras (...) un proceso de civilización concebido empero como de desnaturalización del hombre" (Martínez, p. 180).

Dicha corriente concebía la "cultura" como un fenómeno íntimo y espiritual. Esto derivó en una elegía a la cultura popular, tenida como verdadera, y contrapuesta a la "alta cultura" (relacionada con la "buena crianza”) de las clases gobernantes:

La primera (...) concibe las objetivaciones culturales como una realidad que se cultiva y se adquiere mediante un esfuerzo de refinamiento y un aprendizaje específico (...); la segunda, en cambio, interpreta la cultura como un hecho 
difuso que surge por generación espontánea en la vida social de un (...) grupo (definido por el sexo, la edad, la ubicación social u otras características que implican una esencia), y que se transmite informalmente o se absorbe por ósmosis. En las versiones más esencialistas, se nace con ella o se lleva en la sangre. (Martínez, 1997, p. 185)

El cuadro 1 resume las dos definiciones:

\section{Cuadro 1 Rasgos distintivos de los sentidos amplio y restringido de la cultura}

\begin{tabular}{|l|l|}
\hline $\begin{array}{l}\text { CULTURA } \\
\text { (en sentido amplio) }\end{array}$ & $\begin{array}{l}\text { CULTURA } \\
\text { (en sentido restringido) }\end{array}$ \\
\hline Adscripción & Adquisición \\
\hline Particularismo & Universalismo \\
\hline Difusividad & Especificidad \\
\hline Afectividad & Neutralidad afectiva \\
\hline Orientación a la colectividad & Auto orientación \\
\hline
\end{tabular}

Fuente: Martínez, 1997, p. 187.

En el sentido amplio, "cultura" indica pertenencia a un colectivo con rasgos diferentes y afectuosos. La adquisición emana de un grupo de personas cuya identidad es compartida y distintiva. Para "ser culto" no se requiere de un esfuerzo consciente. Por el contrario, el sentido restringido de "cultura" indica la adquisición de conocimientos y habilidades específicas acerca de grandes temas universales, sin vinculación emocional o identitaria con un grupo particular; de modo individual, se habla de "tener cultura".

Este debate semántico también está presenta en Costa Rica, por lo que el Ministerio de Cultura debe manejar ambos discursos. Por un lado, el Instituto Nacional de la Música y el Museo de Arte Costarricense, cuyos fondos están garantizados por ley, muestran un enfoque más cercano a la acepción restringida de cultura, universalista y académico. Al mismo tiempo, iniciativas como los "Puntos de Cultura" y los "Portadores de Tradiciones", se decantan por el rescate del arte popular como transmisor de identidad más local, en el sentido antropológico del concepto. Así entonces, la gestión cultural costarricense debe asumir dos frentes, a veces por separado, y otras de manera simultánea. Además de ello, y en suelo patrio, se utiliza cada vez más la visión de "cultura empresarial" que abarca el conjunto identitario de valores compartidos, la gestión del conocimiento y los capitales intelectuales (Franklin y Krieger, 2011); la cual es gestionada estratégicamente con el propósito de afianzar la lealtad de los colaboradores y potenciar el talento humano.

\subsection{Patrimonio}

La oficialidad de la cultura, representada por el Ministerio de Cultura y Juventud, utiliza las convenciones de la UNESCO para el patrimonio: cultural, natural, e inmaterial. De hecho, dedican el 25\% de la infraestructura para protegerlo (Monge, 2011). Muchos otros grupos que trabajan con cultura utilizan conceptos alternos o simplemente los adaptan a la cotidianidad. Por eso, vale la pena una revisión crítica, adecuada a la realidad costarricense:

\subsubsection{Patrimonio natural}

Se refiere a sitios, zonas y formaciones; silvestres, geológicas y/o estructurales, de valor global; ya sea por mérito de lo estético o lo científico. Además, Costa Rica cuenta con un $26,21 \%$ de superficie dedicada a reservas naturales protegidas (Monge, 2011). Debido a ello, es imposible separar el ambiente de la cultura.

\subsubsection{Patrimonio cultural}

UNESCO describe el patrimonio cultural como monumentos, conjuntos o lugares "que tengan un valor universal excepcional desde el punto de vista de la historia del arte o de la ciencia" (UNESCO, 1972). La base de datos de Orígenes (2017), registró un total de 4000 sitios arqueológicos en suelo patrio, distribuidos por todo el territorio nacional; los cuales incluyen monumentos, reliquias, construcciones y lugares (Monge, 2011). Esta definición ofrecida a continuación fue complementada más adelante, para incluir el patrimonio inmaterial. 
Se entiende por "patrimonio cultural inmaterial", los usos, íconos, expresiones, conocimientos y técnicas -junto con los instrumentos, objetos, artefactos y espacios culturales inherentes- que las comunidades, grupos y, en algunos casos, individuos, reconocen como pieza integrante de ese legado.Se denomina inmaterial cuando se difunde generacionalmente, siendo recreado siempre por aquellas comunidades y grupos en aras del entorno, la interacción con la naturaleza y la historia; y al infundirles un sentimiento identitario y de permanencia. Contribuyendo también a estimular el respeto por la diversidad cultural y el arte humano (UNESCO, 2003).

\subsubsection{Revisión crítica y propuesta de clasificación}

Las convenciones de UNESCO ofrecen una clasificación del patrimonio cultural muy amplia e inclusiva. No obstante, presentan contradicciones entre sí, lo cual genera zonas oscuras que merecen ser revisadas. En 1972, se describía el patrimonio universal como objetos materiales tangibles. Cuando se amplía el término, en 2003, se incluyen una serie de elementos intangibles ("usos, íconos, expresiones, conocimientos y técnicas"), al mismo tiempo que los objetos materiales relacionados. La ambigüedad es evidente cuando se cuestiona si el patrimonio cultural inmaterial puede ser tangible e intangible a la vez. La cualidad de "inmaterial" está ligada a la transmisión generacional, no a las manifestaciones en sí. Además, en la convención de 1972, se nota una visión restringida de cultura, cuando indica que el valor del patrimonio cultural debe tener carácter universal y ser determinado desde la ciencia y la historia del arte. Por el contrario, según la convención de 2003, el reconocimiento del patrimonio inmaterial proviene de las comunidades o los grupos sociales: Este enfoque, posterior y más contemporáneo, se acerca al sentido amplio de cultura.

Debido a que en Costa Rica coexisten ambos sentidos del término cultura, es necesaria una nomenclatura incluyente, a la vez que distintiva. Debe servir como instrumento de catalogación, a partir de cualidades inherentes a los objetos. Por lo anterior, se propone el siguiente orden taxonómico de una nueva definición adaptada de autoría propia:

\section{3·3.3.1. Bienes culturales tangibles:}

Conjunto de bienes físicos percibidos como valiosos por las comunidades, los colectivos sociales y en algunos casos los individuos; que por sus cualidades funcionales y simbólicas, les infunden sentimientos de respeto, origen, continuidad y trascendencia, verbigracia, antigüedades, instrumentos, artesanías, herramientas, monumentos arquitectónicos, obras de arte, piezas arqueológicas, es decir, objetos que han sido creados o intervenidos por el ser humano.

\subsubsection{Bienes culturales intangibles:}

Son aquellos usos, figuras, expresiones, conocimientos y técnicas que pueblos, colectivos sociales y/o individuos identifican como parte del acervo cultural. Son recreados constantemente por los dueños en función del entorno, la interacción con la naturaleza y la historia; al transmitirles sentimientos de respeto, origen, continuidad y transcendencia.

\section{Una aproximación teórica}

En este punto, ha quedado establecido que la administración clásica evolucionó desde una visión mecanicista hacia una más orgánica y estratégica llamada gestión. También se ha reseñado la existencia de dos visiones de cultura, una restringida y otra amplia, ambas presentes en las convenciones internacionales y en el entorno costarricense. Con fines utilitarios, se ha propuesto una nomenclatura que categoriza los bienes culturales a partir de sus cualidades inherentes. Lo anterior permite analizar la gestión cultural como una práctica profesional relacionada con dichos bienes.

Martinell (2001) explica que la Gestión Cultural no es una ciencia en sí misma; ya que no posee un cuadro de saber único; sin embargo, tiene un eje multidisciplinar. Aun así, la hechura del marco teórico y conceptual debe ser ideada por los propios ejecutores; pues es fruto de la labor de una sociedad en busca de profesionales cumplidores de hartos requisitos. También, es descrita como un complejo fenómeno social, ajustado a un grupo de razonamientos y destrezas provenientes de otras disciplinas. La Gestión 
Cultural "se aplica en un territorio determinado y procura la gestión estratégica, justa y equitativa de los recursos culturales con los que se cuenta en ese espacio específico" (Muñoz, 2009, p.9). Por otro lado, el territorio y la estrategia se suman a la receta, como ingredientes vitales.

\subsection{Ejes temáticos de la Gestión Cultural}

Rodríguez y Protti (2009) han dictado los ejes temáticos y ejecutables que la Gestión Cultural debe añadir a la realidad costarricense. El primero enuncia que la cultura es un elemento potenciador del desarrollo socioeconómico de un territorio, región o país. Esto solo es posible cuando los elementos administrativos, financieros y cuantitativos son dispuestos para permitir la "sostenibilidad política, creativa y esencialmente humana" (Rodríguez y Protti, 2009). Para el desarrollo nacional, es imprescindible ejecutar un examen vigente y cuantitativo del legado cultural; todo ello puede llevarse a cabo al instituir una adecuada guía de las políticas en materia de cultura. Asimismo, la satisfacción de las necesidades ciudadanas es una tarea que ha sido transferida gradualmente desde lo público hasta lo privado. En consecuencia, el ámbito mixto y la cooperación intersectorial deben ser fortalecidos.

Por otro lado, el segundo eje es la diversidad cultural, donde los autores oponen la diversidad ante los efectos masificados de la globalización. Para estos, la identidad se construye a partir procesos sociales e imaginarios colectivos, al contrario de la programación masiva y estandarizada de los grandes medios del entretenimiento. Por ende, el respeto a las minorías, junto con las modas estilísticas clásicas, tradicionales, urbanas y contemporáneas, deben ser asunto diario de una gestión cultural responsable.

Finalmente, el último eje es la economía de la cultura. En ella hay grandes incógnitas sobre el impacto que el acervo cultural tiene en el desarrollo nacional; por tanto, urge un análisis que permita una planificación adecuada. Los estudiosos anteriormente citados sugieren "proyectos ligados a los conceptos de desarrollo, diversidad, leyes y políticas para la cultura" (Rodríguez y Protti, 2009, p.33). Así también, enumeran además varios protagonistas como: el turismo, las industrias y el patrimonio cultural e incluso los mercados del arte. El primero generaría riqueza en el nivel local y un mayor entendimiento del mundo, mientras que las segundas constituirían una "importante fuente de empleo, generación de divisas, potencial estímulo a la creatividad, impulso a la innovación de procesos productivos y de distribución y promotoras de bienes y servicios culturales" (Rodríguez y Protti, 2009, p.32). Los cambios tecnológicos imponen necesidades distintas en la producción y en la logística. El comportamiento de los pueblos adquiere valor destacado, pues hay una mayor demanda de símbolos, valores y significados como bienes de consumo a gran escala. Por otra parte, la complejidad de la producción y el consumo cultural no pueden ser medidos por indicadores de la administración dura. Es misión de la gestión cultural proponer métodos adecuados de evaluación.

\section{Una aproximación histórica}

La forma de la Gestión Cultural ha presentado un estrecho nexo con las políticas nacionales y extranjeras, las cuales se insertan en el conjunto amplio de la planificación del estado. Por eso, y para concebir la estructura presente del sector cultural, es necesario un breve atisbo holístico al desarrollo de la república.

Las primeras políticas culturales de Costa Rica inician en el siglo XIX. El auge de las exportaciones permitió a la aventajada clase dirigente establecer intercambio con países de Europa; ello trajo una seguridad financiera patrocinadora del arte (Brenes, Quesada y Sobrado, 1997). Más tarde, las primeras ideas se centraron en la génesis de una cultura basada en los grandes artistas y pensadores del "viejo continente", es decir, las primeras instituciones de cultura costarricense fueron "europeístas" (Ferrero, 1986).

A finales del siglo XIX (1887), fueron inaugurados el Museo Nacional, la Escuela Nacional de Bellas Artes y el Teatro Nacional. El primero reunió un acervo de "productos naturales y curiosidades históricas y arqueológicas" (Sistema Costarricense de Información Judicial, s/f). La segunda y el tercero se dedicaron a importar y reproducir obras clásicas, con el respaldo del apoyo monetario de la clase cafetalera, y como "certificados" de estatus social 
(Rovinsky, 2006). Esta manía de “consumo cultural”, bajo el entendido de "educación refinada", perduró décadas como privilegio de una clase educada, residente en las capitales de provincia (Ferrero, 1986).

Durante el siglo XX, Costa Rica adoptó dos corrientes de pensamiento político, influidas por los escenarios mundiales. Entre las décadas de 1940 y 1970, se asentó un modelo gubernamental proteccionista: el Gobierno era responsable de satisfacer todas las necesidades de los ciudadanos. Para los ochentas, las relaciones económicas mundiales sufrieron giros importantes que cerraron con el declive del llamado "bloque soviético", y con este, la influencia social del estado paternalista perdió credibilidad. A partir de 1980, las políticas neoliberales reemplazaron a las anteriores. El cambio promovió el libre comercio, la mínima interferencia del Estado en la economía nacional, el levantamiento de barreras arancelarias y la globalización de los mercados.

Debido a la carga impuesta por organismos foráneos, se modificó el estilo administrativo; ello trajo un descenso en la atención de las necesidades del pueblo, debilitó los mercados nacionales, atrayendo la inversión extranjera. Las reformas estructurales en el país y el desvanecimiento del "proteccionismo" fortalecieron las organizaciones civiles independientes, las cuales debían alcanzar la sostenibilidad por sus propios medios. Se puede decir que "hay un proceso de "des-gubernamentalización", evidente en el retiro del "Estado Benefactor", y su reemplazo por instituciones heterogéneas y más "microgerenciadas" por la sociedad civil” (Rodríguez y Protti, 2009).

En el plano internacional, se dio una nueva revisión de la cultura con el fin de otorgarle un protagonismo en el desarrollo social y económico. Los paradigmas de "progreso" fueron sustituidos por el "desarrollo humano integral", y se agregaron los "indicadores culturales de crecimiento económico":

Esta etapa de "institucionalización" implicó dos aspectos elementales: primero, la nueva potestad adquirida por el Gobierno para fijar códigos y jerarquías a los valores y significados de la cultura; y por el otro, que asumiera este también el papel de centralizar y unificar las acciones que competen a la cultura, a través del establecimiento de instituciones y agentes. (García, 2011, p. 3).

En Costa Rica, en la década de 1970, se creó el Ministerio de Cultura, Juventud y Deportes, el cual dispensaba en un principio una visión "eurocentrista" de la cultura, conocida como "alta cultura" (Ministerio de Cultura y Juventud de Costa Rica, Archivo Central, 2016). Ahora bien, mientras los acuerdos internacionales le imponían la prestación de servicios culturales, se exigía simultáneamente un fuerte descenso del aparato estatal. Por otro lado, una óptica más amplia del arte, tendencias mundiales a la oferta y venta de recursos, así como el desarrollo de mercados culturales, provocaron que iniciativas privadas se consolidaran, tanto en el nivel de república como local (Martinell, 2009). Todo ello impulsó las alianzas entre el Ministerio y entes privados, y el surgimiento de organizaciones mixtas.

Cabe agregar que la reducción del apoyo del Gobierno dibujó un panorama latinoamericano, en el cual, los territorios vecinos, junto con distintos agentes sociales, han generado demandas de desarrollo socioeconómico para el sector cultural. Los gobiernos legitimaron dichas peticiones mediante el planteamiento de políticas internas. Lo anterior, liberó un proceso de generación de servicios, equipamientos y actividades; forjadores todos de un campo de actividad novedoso, el cual ha llegado a denominarse Gestión Cultural, y que tomó relevancia y protagonismo en todos los ámbitos socioeconómicos (Martinell, 2009).

En 2013, tras varios años de diagnóstico y planificación, se editó la Política Nacional de Derechos Culturales 20142023, cuya fase de planteamiento: "Este proceso permitió identificar las necesidades más apremiantes que existen en materia cultural en el contexto actual costarricense y, además, los aspectos que deberán recibir una atención prioritaria por parte del Estado durante el próximo decenio" (Ministerio de Cultura y Juventud, 2013, p.6). Ello constituyó un marco político definido para el régimen del Estado orientado en 5 ejes estratégicos:

1. Participación efectiva y disfrute de los derechos culturales en la diversidad. 
2. Dinamización económica de la cultura.

3. Protección y gestión del patrimonio cultural, material e inmaterial.

4. Fortalecimiento institucional para la protección y promoción de los derechos culturales.

5. Derechos culturales de los pueblos indígenas.

\section{Los objetos de la Gestión Cultural costarricense}

Esta disciplina se enfoca en el manejo adecuado de los recursos culturales en grupos, comunidades y organizaciones. En esta definición, esos recursos incluyen capitales de varios tipos: talento humano, bienes (materiales e inmateriales) y vínculos sociales. El talento humano puede ser identificado en un individuo, una comunidad o una organización. No se refiere a las personas solamente como entes físicos; empero se incluyen las capacidades, las experiencias, la educación y los valores simbólicos, éticos y estéticos. "Para Mathis y Jackson (2008), el capital intelectual refleja el pensamiento o inteligencia, conocimiento, creatividad y toma de decisiones" (Briceño y Godoy, 2012, p. 63). Una organización es una: "entidad que tiene un fin determinado, personas o miembros y una estructura deliberada" (Robbins y Coulter, 2005, p. 16). Cabe destacar, que las organizaciones no son estáticas; sino que van cambiando en la esencia, tamaño, intereses, configuraciones, estructuras y valores. Están vivas en los tres sectores del quehacer socioeconómico: público, privado y mixto. Los bienes culturales, previamente descritos, se dividen en tangibles e intangibles.

Las relaciones sociales abarcan aquellas acaecidas entre los seres humanos y los congéneres, y las desarrolladas con el entorno. Esto implica dinámicas entre los humanos con otras formas de vida (animales, plantas, ecosistemas) y con objetos inanimados; así como los vínculos entre colectivos (entidades, comunidades, regiones, países). Son un recurso cultural en la medida que, siendo intangibles, afectan el funcionamiento de los procesos sociales y son protagonistas, cuando se trata de articular esfuerzos desde diferentes aristas con sendos intereses.

\section{Práctica profesional}

Ahora bien, a partir de la consulta realizada a gestores culturales en el 2014 (ver metodología), quienes ejercían de manera empírica, se ha elaborado este cuadro, el cual evidencia un resumen comparativo de las prácticas profesionales relacionadas con la gestión. Las prácticas decadentes son aquellas que han caído en desuso, a pesar de persistir de forma incipiente. Las prácticas dominantes son las más comunes en la actualidad, mientras que las prácticas emergentes van tomando un protagonismo cada vez mayor.

\section{Cuadro 2. Prácticas profesionales ejecutadas por gestores culturales costarricenses}

Prácticas Decadentes

Beneficencia a grupos sociales y comunidades. Centralización de la oferta cultural en la Gran Área Metropolitana (GAM).

Imposición de modelos de desarrollo local.
Promoción del arte clásico europeo como cultura oficial. Promoción del folklore guanacasteco como nacional. Proteccionismo estatal. 
Prácticas Dominantes

Administración Pública.

Alienación de la oferta cultural, se toman diseños artesanales para producirlos en masa, en el extranjero, y venderlos en tiendas de recuerdos.

Animación cultural.

Articulación interinstitucional.

Bienestar y desarrollo social.

Conservación de los patrimonios natural y cultural tangible.

Cooperación internacional.

Creación y Gestión de Pequeñas y Medianas Empresas (PYMES).
Defensa de los Derechos Humanos (DD.HH.).

Docencia y capacitación.

Formulación, Gerencia y Evaluación de Proyectos.

Gestión Ambiental.

Gestión del Talento Humano.

Gestión Local.

Masificación de la oferta cultural, el (la) gestor(a) trabaja en medios de comunicación masiva o para grandes eventos internacionales.

Museología.

Organización de Eventos.

Prácticas Emergentes

Capitalización del conocimiento y la creatividad. Conservación del patrimonio cultural intangible. Democratización de la información.

Desarrollo de la creatividad en las organizaciones.

Evaluación social de proyectos.

Formación de líderes locales.

Gestión de la Cultura Organizacional.

Gestión de Redes Sociales.

Investigación de mercados culturales.
Gestión del conocimiento.

Mapeo cultural de comunidades.

Mediación con grupos de interesados.

Motivación de los colaboradores.

Organización de eventos de diseño y creatividad.

Responsabilidad Social Empresarial.

Servicios internacionales a clientes y empresas.

Teletrabajo.

Turismo cultural y rural.

Fuente: elaboración propia a partir de la consulta realizada a gestores culturales.

El panorama anterior exhibe cómo las prácticas decadentes se relacionan con la idea de "alta cultura" y de "Estado proteccionista”. Las entidades del Gobierno, culturales y educativas, imponían manifestaciones tradicionales: música clásica, literatura griega y española, y artes plásticas figurativas. Además, se daba un aglutinamiento de esta receta en los centros urbanos de San José, Alajuela, Heredia y Cartago. La construcción de los mayores teatros, museos y bibliotecas en estas ciudades es una prueba fidedigna.

Las prácticas dominantes han surgido a partir de los cambios en la estructura económica y política del orbe. La reducción del aparato estatal, de enfoque neoliberal, ha implicado que organizaciones civiles asuman la oferta de servicios públicos, el disfrute de los derechos culturales entre ellos. Esto ha impulsado el vigor de la estrategia comunitaria y el crecimiento de un ámbito socio productivo mixto y solidario. Las entidades son más autogestionarias y menos dependientes del Estado. Se ha impuesto la coyuntura entre grupos, en todos los ámbitos; así como la ayuda exterior. El descarte de barreras arancelarias ha atraído capital extranjero, multinacionales y turismo cultural con matices rurales. Las tecnologías de la información y la comunicación (TIC) se han incorporado en la Gestión Cultural, tal es el caso del teletrabajo, los derechos de propiedad intelectual, el mercadeo y las redes sociales. Así también, se ha ejecutado el Emprendedurismo, un modelo aplicado al sector cultura en pequeña y mediana escala. También son comunes muchos de los temas vinculados a la cultura empresarial: asesoría, gerencia de proyectos, gestión del conocimiento, "empowerment" (o empoderamiento de los colaboradores) y responsabilidad social de empresa (RSE).

Por su parte, las prácticas emergentes se vuelcan hacia la planificación estratégica, la formación de líderes locales, el mercadeo, la creatividad metodológica y la 
internacionalización. A su vez, las redes sociales en plataformas de la Internet, el premio al ingenio y la novedad, el mapeo de actores y la animación cultural, son elementos recientes, pero que van afirmándose en todo tipo de organizaciones.

\section{Reflexiones finales}

La definición de la cultura es un trabajo de permanente reconstrucción. Si bien las convenciones internacionales han brindado un marco estandarizado, que permite un lenguaje común; es fundamental el ajuste con las realidades particulares de los fenómenos nacionales y locales. Por otro lado, "patrimonio intangible, natural y/o arquitectónico" son usos no ligados a hechos de vanguardia, por lo que se propone una reforma a esos conceptos.

Ahora bien, la diversidad cultural se perfila necesaria ante la homogeneidad de los mercados, el resguardo de los derechos individuales, el amparo a los grupos frágiles y la merma del ingenio. ¿De dónde, pues, se puede evolucionar, si no es desde el cambio? La Política Nacional de Derechos Culturales 2014-2023 indica que el Estado honra y abraza lo diverso en la estructura socioeconómica. En tanto, incapaz de satisfacer los menesteres culturales de la gente, ha decidido volcar los ánimos en pro de las organizaciones civiles, al juzgar lo tradicional como motor del progreso.

Asimismo, se expresa un interés por lo autóctono y las muestras artísticas, a priori invisibilizadas. Lo mismo sucede en el campo del derecho, no obstante, la normativa sobre el tema ha sido puesta al día con lentitud. Por el contrario, permanecen organismos herederos del "Estado Protector” los cuales, blindados con garantías legales, se hallan anclados en el sentido restringido de cultura, la cual diviniza los modelos eurocéntricos de arte y filosofía, cuyo entendimiento requiere de una educación formal y clásica, distante de la realidad de la mayoría de los costarricenses.

Cabe agregar que, Costa Rica tiene un patrón organizativo capitalista dividido en ámbitos (público, privado y mixto) y la Gestión se desplaza a lo largo de ellos. La cultura es un motor de desarrollo social, sobre todo en una época, cuyos límites políticos se desdibujan ante la virtualización y las franquicias. Su carácter complejo y variable requiere metodologías equivalentes, las cuales no pueden ser rígidas. Bajo estos términos, la Gestión utiliza las bases administrativas, pero debe adaptarlas un entorno cambiante, de forma espontánea e ingeniosa, pero estructurada. En Costa Rica, las personas que hacen gestión cultural se han vuelto profesionales muy específicos, ajustados al entorno, quienes deben poseer una amplia gama de conocimientos y técnicas en: filosofía, arte, ciencia, tecnología, análisis, información, política y estrategia comunitaria, etc.

En fin, la Gestión Cultural es más ardua de lo que parece; esto a razón de la atmósfera política y de la sociedad en boga; pues no se trata solo del cabildeo público; sino de interconexiones pluridisciplinarias y del empleo de numerosas aptitudes. Incluso si hombres y mujeres de buena fe encararon dicha práctica ab illo tempore, ha llegado la hora de que profesionales especializados viren el timón de este barco y lo lleven a nuevos destinos.

\section{Bibliografía}

Alpízar, N., et altri. (2014). Mercados culturales en Costa Rica: un diagnóstico previo a la creación de la carrera de Gestión Cultural en la Universidad de Costa Rica. Universidad de Costa Rica, Sede del Pacífico.

Bolaños, C. (s.f.) Elementos de la propuesta curricular, Centro de Evaluación Académica, Universidad de Costa Rica, recuperado de http://www.cea. ucr.ac.cr/media/diea/publicaciones/esquema_ diseno_curricular.doc

Brenes, M. V., Quesada, L. G. B., y A. M. B. Sobrado. (1997). Historia de Costa Rica: hacia la formación del Estado, 1821-1870. San José, Costa Rica: EIDOS.

Briceño, M., y F. Godoy. (2012). El Talento Humano: un capital intangible que otorga valor a las organizaciones. En International Journal of Good Conscience. Abril, 7(1), 57-67. 
Buitrago, F., y I. Duque. (2013). La economía naranja: una oportunidad infinita. [Documento electrónico]. Recuperado de: https://publications.iadb.org/ bitstream/handle/11319/3659/La economía naranja: una oportunidad infinita.pdf

Chiavenato, I. (2006). Introducción a la teoría general de la administración. Mc Graw Hill Interamericana. México.

Drucker, P. F. (1994). The Post-Capitalist Society. [Documento electrónico]. Recuperado de: https:// doi.org/10.1016/B978-0-7506-0921-0.50001-X

Ferrero, L. (1986). Sociedad y arte en la Costa Rica del siglo XIX. San José, Costa Rica: EUNED.

Franklin, E., y M. J. Krieger. (2011). Comportamiento organizacional: enfoque para América Latina. México: Pearson Education. García Román, L. E. (2011). Una revisión teórica sobre la Gestión Cultural. En Revista digital de Gestión Cultural, 1(1), 5-15. [Documento electrónico]. Recuperado de: http://gestioncultural.org.mx/revista/o1/ Elena_Roman_N1_A1.pdf

García Román, L. E. (2011). Una revisión teórica sobre la gestión cultural. Revista Digital de Gestión Cultural, 1(1), 5-15. [Documento electrónico]. Recuperado de: http://gestioncultural.org.mx/ revista/o1/Elena_Roman_N1_A1.pdf

González Jiménez, L., y A. Hernández Sánchez. (2012). Producción cultural en Costa Rica. En Estado de la Nación, 18-52. [Documento electrónico]. Recuperado de: http://www.estadonacion.or.cr/ files/biblioteca_virtual/o18/gonzalezjimenez_y_ hernandez_produccion_cultural.pdf

Martinell, A. (2007). La gestión cultural: singularidad profesional y perspectivas de futuro (recopilación de textos). En Seminario internacional: la formación en gestión y políticas culturales para la diversidad cultural y el desarrollo, 23-46. [Documento electrónico]. Recuperado de http:// www.cidadeimaginaria.org/gc/GCprofut.pdf
Martinell, A. (2009). Las interacciones en la profesionalización en Gestión Cultural. En Pensamiento Iberoamericano, 4, 277-288.

Martínez Sahuquillo, I. (s/f). Los dos conceptos de cultura: entre la oposición y la confusión. En Revista Española de Investigaciones Sociológicas (REIS), 173-196.

Ministerio de Cultura y Juventud de Costa Rica. (2013). Política Nacional de Derechos Culturales, 20142023. San José, Costa Rica: MCJCR.

Ministerio de Cultura y Juventud de Costa Rica, Archivo Central. (2016). Entrada descriptiva con la aplicación de la norma internacional ISAD (G) [documento electrónico]. Recuperado de: http://mcj.go.cr/ministerio/archivo_central/ documentos_archivo/ISADFondoMCJ.pdf

Monge, M. J. (2011). Diagnóstico de la situación de la cultura en Costa Rica. San José, Costa Rica: MCJCR.

Muñoz, M. (2009). Capacitación de artistas y gestores culturales en desarrollo local e interculturalidad. San José, Costa Rica: MCJCR.

Robbins, S. P., y M. Coulter. (2005). Administración (8 ${ }^{\mathrm{a}}$ Ed.). México: Pearson Education.

Rodríguez, E., y G. Protti. (2009). Diagnóstico para la formación en Gestión Cultural en Costa Rica. Asociación cultural Giratablas. San José, Costa Rica.

Rovinsky, Y. (2006). El Teatro Nacional de Costa Rica: su historia (resumen del libro de Astrid Fischel). [Documento electrónico]. Recuperado de: https:// tnwebdata.teatronacional.go.cr/repositorio/ detail/28-1875_resumen_historia_teatro_ nacional_costarica.pdf 
Sistema Costarricense de Información Judicial. (s/f). Ley Orgánica del Museo Nacional. [Documento electrónico]. Recuperado de: http://www.pgrweb.go.cr/scij/Busqueda/ Normativa/Normas/nrm_texto_completo. aspxparam $1=$ NRTC $\&$ nValor $1=1 \&$ nValor $2=$ $68 \&$ nValor $3=70 \&$ strTipM $=\mathrm{TC}$

Smith, A. (1776). La riqueza de las naciones (traducido por Carlos Rodríguez Braun, en 1996). Primera edición, El libro de bolsillo (colección de Ciencias Sociales). Editorial Alianza, Madrid, España.

UNESCO (1972) Convención sobre la protección del patrimonio mundial, cultural y natural. [Documento electrónico]. Recuperado de: https:// whc.unesco.org/archive/convention-es.pdf

UNESCO. (2003). The impact of uniform laws on the protection of cultural heritage and the preservation of cultural heritage in the 21st century. En Convention for the safeguarding of the intangible cultural heritage. [Documento electrónico]. Recuperado de: https://doi. org/10.1163/ej.9789004180444.I-786.6 